\title{
EFFECTS OF ADRENORECEPTOR ACTIVATION AND AGING ON SKELETAL MUSCLE ARTERIOLES AT REST AND DURING RAPID ONSET VASODILATION
}

\author{
Alex W. Moore \\ Dr. Steven S. Segal, Dissertation Supervisor
}

\begin{abstract}
Sympathetic nerve activity (SNA) induces arteriolar vasoconstriction via $\alpha$ adrenoreceptor ( $\alpha A R$ ) activation. Whether $\alpha A R$ activation affects the spread of rapid onset vasodilation (ROV) in contracting muscle is unknown. Differential $\alpha A R$ distribution in vascular smooth muscle has been proposed to mediate functional sympatholysis, however the $\alpha A R$ subtype distribution in locomotor muscle is undefined. This dissertation determined: 1 ) the effects of constitutive $\alpha A R$ activation on the spread of ROV within contracting muscle, 2) the functional $\alpha A R$ distribution in locomotor muscle of the mouse, and 3) the influence of $\alpha A R$ on ROV during aging. In arterioles of the gluteus maximus muscle (GM), I tested the hypotheses that: 1) adrenoreceptor subtype distribution is heterogeneous and 2) adrenoreceptor activation modulates the spread of ROV. The left GM of young (3-month) anesthetized C57BL/6 mice were studied using intravital microscopy. Distinct anastomotic, 1A, 2A, and 3A arterioles were studied at rest and following single muscle contraction in the presence or absence of topical $\alpha A R$ agonists and antagonists. Functional $\alpha A R$ distribution differed between proximal and distal arterioles. Constitutive $\alpha A R$ activation inhibited the spread of ROV between regions of the GM. It also reduced the amount of ROV seen in old ( 20 -month) versus young male mice. I conclude that functional $\alpha A R$ are heterogeneously distributed in arteriolar networks and serve to modulate regional vasodilation.
\end{abstract}

